\title{
The resurgence of Measles in 2019
}

Sir,

Measles, a highly contagious infection caused by a single stranded RNA Paromyovirus is transmitted through aerosol droplets and contact with nasopharyngeal secretions of the infected patient. The incubation period lasts between 10 and 14 days. The Prodromal phase presents with high grade fever, cough, coryza and conjunctivitis. Punctate blue-white spots on the buccal mucosa called Koplik spots are pathognomic of measles. The typical maculopapular rash appears 3-5 days after the onset of fever. It starts from the neck and spreads down to the lower limbs; it decreases in the same pattern lasting for 5-6 days. The patients are considered contagious 4 days prior to 4 days after the appearance of rash.

Transient immunosuppression during the acute and convalescent phases can lead to diarrheal episodes, secondary viral and bacterial pneumonias. Other complications include blindness, deafness and CNS pathologies like primary measles encephalitis (PME), acute disseminated encephalomyelitis (ADEM), measles inclusion body encephalitis (MIBE), and the rare, fatal subacute sclerosing panencephalitis (SSPE). Protein energy malnutrition, Vitamin A deficiency, immunodeficiency and vaccination status determine the increased morbidity and mortality due to measles. Fatality rates are higher in the developing countries when compared to developed nations. Inspite of the availability of an effective and safe vaccine, which has been included in the national immunization schedule of most countries, measles still kills more children than any other vaccinepreventable disease.

Basic reproduction rate (R0), a measure of the communicability of a pathogen, is defined as the average number of secondary cases infected by a single primary case in a susceptible non immune population. The $R(0)$ of measles is 12-18, making it the most infectious disease known. ${ }^{1}$ A herd immunity level of $90 \%-95 \%$ is required to stop outbreaks and achieve endemic disease elimination. ${ }^{2}$ The high transmissibility of measles, and detection of cases, is helpful in identifying gaps in vaccination coverage and hence it is considered the sensitive "canary in the coal mine", in public health.

The first decade of the new millennium (2000-2010) saw good progress with a $74 \%$ decrease in measles mortality globally; due to sustained mass national vaccination programs with the WHO recommended 2 dose schedule. ${ }^{3}$ Elimination of endemic measles was achieved in the Americas in 2002, which lasted for more than a decade. ${ }^{4}$
The Western pacific region and China reported more than $90 \%$ coverage of the two dose vaccine schedule. ${ }^{5}$

The progress has since stagnated in the present decade. A worrisome resurgence of measles has been ongoing globally for the past few years. Canada, Brazil and the USA have been experiencing outbreaks since 2014, mostly because of cases brought into the country by nonimmune travellers from endemic areas. Continuing outbreaks in Africa, Israel, China, Philippines, Ukraine and Europe have also been reported. ${ }^{6}$ The Global Vaccine Action Plan, 2012, had targets to achieve endemic measles elimination in 5 WHO regions by 2020 , which probably will not be met.

The United States has seen a recent uptick in the number of diagnosed cases. Between January and June 2019, the CDC has reported 1095 cases in 28 of the 50 states. $^{7}$ This is considered the highest number of measles cases reported in the past 25 years and since it was declared eliminated in 2000. The initial outbreaks were detected in New York and Washington, where it was confined to few close-knit communities with unvaccinated individuals.

Vaccine Hesitancy and loopholes in the law which allow parents to refuse vaccinations have been the main reasons for measles to gain back a foothold in the USA. The enormous influence of social media and constant misinformation circulated by the anti-vaccination campaign groups has resulted in a steady drop of vaccination coverage, in spite of easily available vaccination services, all across the globe.

Pediatricians and Family Medicine specialists are the front-line healthcare workers who need to dispel the myths around vaccination and make sure that the child gets immunizations done at the right time. The governments and World health organization should also play a critical role in tailoring a strict vaccination policy, educating the masses, and thus reduce the risk of outbreaks because of vaccine hesitancy. Social media giants like Facebook and Google need to do their part by taking down websites and articles promoting misinformation about vaccination.

The tremendous historical achievements made in the field of public health in reducing the prevalence of infectious diseases are being threatened by the spread of scientifically non credible information. An urgent collaboration is required between health care workers, government agencies, World Health Organization and social media platforms to tackle this issue and ensure that 
the future generations are not at an increased risk from vaccine preventable diseases.

\section{Chaitanya Varma P. V.*, Samitha Rajkumar}

Department of Pediatrics, Unicare Medical Centre. Dubai, UAE

\section{*Correspondence:}

Dr. Chaitanya Varma P. V., E-mail: pvcvonline@gmail.com

\section{REFERENCES}

1. Kelly HA, Riddell MA, Andrews RM. Measles transmission in healthcare settings in Australia. Med J Aust. 2002;176(2):50-1.

2. Orenstein WA, Gay NJ. The theory of measles elimination: implications for the design of elimination strategies. $\mathrm{J}$ Infect Dis. 2004;189(S1):S27-35.

3. Simons E, Ferrari M, Fricks J, Wannemuehler K, Anand A, Burton A, et al. Assess-ment of the 2010 global measles mortality reduction goal: results from a model of su rveillance data. Lancet 2012;379(9832):2173-8.

4. Centers for Disease Control and Prevention. Progress in global measles control, 2000-2010. Morb Mortal Wkly Rep. 2012;61(4):73-8.

5. Sniadack DH, Mendoza-Aldana J, Jee Y, Bayutas B, Lorenzo-Mariano KM. Progress and challenges for measles elimination by 2012 in the Western Pacific Region. J Infect Dis. 2011;204(1):S439-46.

6. World Health Organization Western Pacific Region. Measles - Rubella Bulletin, June 2014; 2014.

7. CDC. Measles cases and outbreaks: measles cases in 2019. Atlanta, GA: US Department of Health and Human Services, CDC; 2019. Available at: https://www.cdc.gov/measles/cases-outbreaks.html

Cite this article as: Varma PVC, Rajkumar S. The resurgence of Measles in 2019. Int J Contemp Pediatr 2019;6:2738-9. 